\title{
Imaging Ice Embedded Single Particles With A 16 Megapixel CCD Camera
}

\author{
Christopher R. Booth ${ }^{1}$, Donghua Chen ${ }^{2}$, Steven J. Ludtke ${ }^{2}$, Wah Chiu ${ }^{1,2}$ \\ ${ }^{1}$ Program in Structural and Computational Biology and Molecular Biophysics, ${ }^{2}$ National Center for \\ Macromolecular Imaging, Verna and Marrs McLean Department of Biochemistry and Molecular \\ Biology, Baylor College of Medicine, Houston, Texas 77030, USA
}

Charge coupled device (CCD) cameras have been widely used in electron microscopy. The modulation transfer function (MTF) of CCD cameras and their smaller imaging area relative to film have limited their routine use for high resolution data collection of ice embedded biological specimens [1]. However, the performance specifications of the current generation of 16 Megapixel (4096x4096 pixels) CCD cameras (GatanUS4000) appear to be feasible for single particle imaging to at least 8-10 $\AA$ resolution [2]. We have evaluated the CCD camera performance on a JEOL2010F electron microscope operated at $200 \mathrm{keV}$.

We initially used the "noise method" [3] to estimate the MTF of the CCD camera (Figure 1A). Secondly we compared the signal to noise ratios (SNR) of amorphous carbon film images acquired on CCD camera and Kodak SO-163 film. Figure 1B shows that the CCD image has higher SNR than the photographic film image below 1/3 Nyquist frequency but not so at higher spatial frequencies.

In order to work with ice embedded specimens, we have developed a library of computer routines called the JEOL Automated Microscopy Expert System (JAMES), which interfaces between Gatan's CCD controlling software (Digital Micrograph), the JEOL FasTEM software, and our database (EMEN). This library is written in both $\mathrm{C}++$ and Python to take advantage of the features of both languages. Once a suitable specimen area is identified and the focus is manually set, JAMES records a focal pair of ice embedded single particle images in the same area at a pre-set low dose, with desirable defocus values. The microscope imaging conditions are then automatically uploaded to our database server along with the newly acquired images. Figure 2A typifies the first CCD image in a focal pair of ice embedded cytoplasmic polyhedrosis virus (CPV) taken at 60,000x magnification. The power spectrum of the particle images shown in Figure 2B shows the contrast transfer function rings visible to $8.6 \AA$. The SNR analysis of this data confirms that $5 \%$ contrast is detectable at $8.6 \AA$, which is equivalent to $5 / 12$ Nyquist frequency. Once the microscope is aligned and an area suitable for imaging is identified, we were able to acquire and upload a focal pair of images every 3 minutes. Using this system, an $8 \AA$ reconstruction of $\mathrm{CPV}$, which is likely to require 5,000 particles ( 200 focal pairs), could be completed in 10 hours of imaging time. Even if we were to double the amount of data needed, the entire data collection process could be finished within 3 days. When used in combination with SAVR, our software for icosahedral particle reconstructions [4], we expect to complete a subnanometer structure within a few weeks.

References

[1] Sherman MB, Brink J, Chiu W. Micron (1996) 27: 129-39.

[2] Gatan Inc. Ultrascan Brochure, (2001).

[3] de Ruijter WJ and Weiss JK. Rev. Sci. Instruments (1992) 63: 4314-4321.

[4] Jiang W, Li Z, Booth CR, Baker ML, Chiu W. J. Struct. Bio. (2001) 136:214-225.

Acknowledgements: We thank Dr. Z.H. Zhou for providing the CPV specimens and J. Jakana for her help. This research is supported by P41RR02250, R21AI053737 and Welch Foundation. 

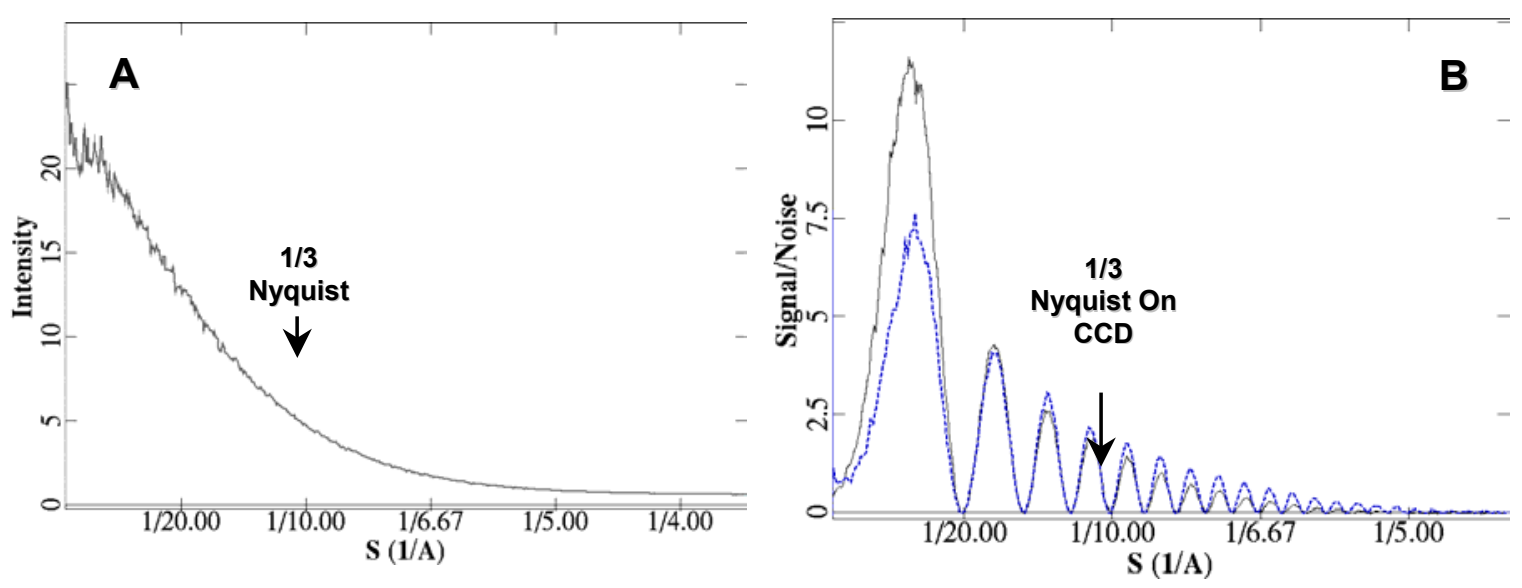

Figure 1: Imaging characteristics of the CCD camera. (A) The MTF of the CCD calculated using the Noise method, showing the attenuation of the signal at higher spatial frequency. (B) A comparison between the SNR from amorphous carbon images by using the CCD or Kodak SO-163 film scanned on a Ziess Phodis SCAI scanner with a $7 \mu \mathrm{m}$ step size. This shows the attenuation of the signal from the CCD at higher spatial frequency in comparison with film.
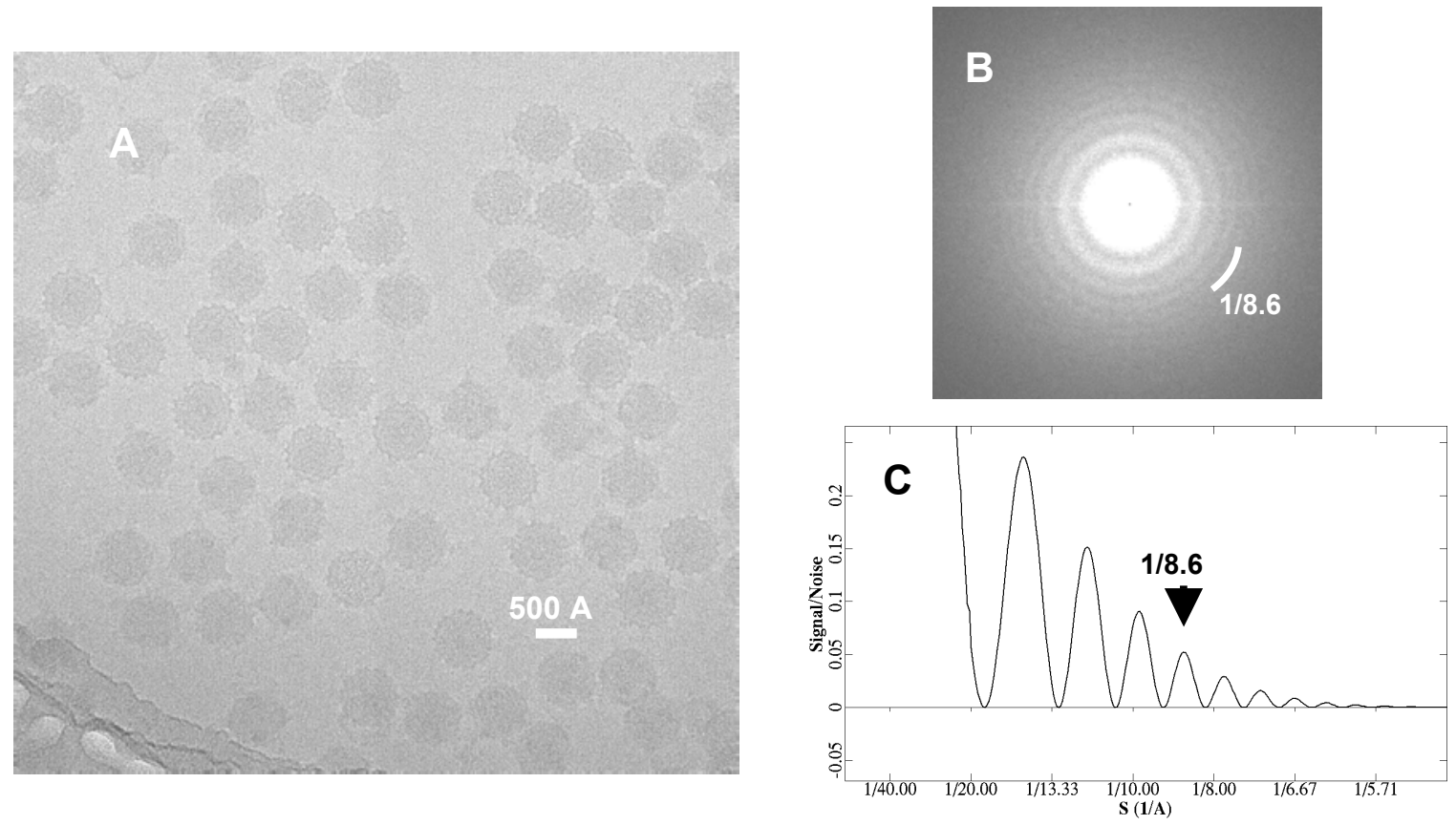

Figure 2: Ice embedded viral particles. (A) First image in a focal pair of ice embedded CPV acquired using the CCD. (B) Power spectrum from approximately 50 ice embedded CPV particles incoherently averaged together. (C) A 1-D SNR calculated from (B). 5\% Signal is clearly detectable beyond $1 / 9 \AA$ resolution. 